International Journal of Pure and Applied Mathematics

Volume 115 No. $3 \quad 2017,467-475$

ISSN: 1311-8080 (printed version); ISSN: 1314-3395 (on-line version)

url: http://www.ijpam.eu

doi: 10.12732/ijpam.v115i3.2

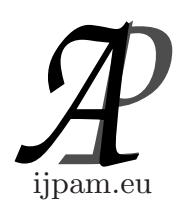

\title{
NUMERICAL SOLUTION OF FRACTIONAL \\ DIFFERENTIAL EQUATION SYSTEM USING THE MÜNTZ-LEGENDRE POLYNOMIALS
}

\author{
Mohammadreza Aghashahi ${ }^{1}$, Mojtaba Rasouli Gandomani ${ }^{2} \S$ \\ ${ }^{1}$ Department of Mathematics \\ Aghigh Institute of Higher Education \\ Shahin Shahr, Isfahan, IRAN \\ ${ }^{2}$ Department of Mathematics \\ Isfahan (Khorasgan) Branch \\ Islamic Azad University \\ Isfahan, IRAN
}

\begin{abstract}
In the paper, we apply a numerical approach to approximate solution of a system of the fractional differential equations based on the Müntz-Legendre polynomials. Features of these polynomials make them so appropriate for solving the fractional differential equation system. In the proposed method, a system of the fractional differential equations is transformed into a system of the algebraic equations which is move easier to solve. The numerical examples show accuracy and convergence of the present method.
\end{abstract}

AMS Subject Classification: 34A08, 35R 11

Key Words: fractional differential equations, Müntz-Legendre polynomials, collocation method

\section{Introduction}

Fractional calculus plays main role in physics, chemistry and engineering which has attracted the interest of many researchers in recent years. In comparison to

Received: October 4, 2016

Revised: $\quad$ May 24, 2017

Published: July 27, 2017

(c) 2017 Academic Publications, Ltd. url: www.acadpubl.eu

$\S_{\text {Correspondence author }}$ 
the differential equations of accurate order, the fractional differential equations (FDEs) have an advantage that many phenomena in physics, chemistry and engineering can be described very successfully by them. Moreover, this allows one to apply the fractional differential equations to model these phenomena more accurately. Some of these models and applications are presented in $[1,2$, $3,4,5]$. Also many researchers tried to investigate and describe these kinds of the equations (see Ref. $[6,7]$ ). Odibat suggested an analytical examination for FDEs [8]. Jafari and Daftardar also proved existence and uniqueness solutions of the FDEs [9]. Obtaining an exact solution of these problems is extremely difficult thus there are a lot of numerical methods to approximate solutions of the FDEs such as, Homotopy method [10], finite differences method [11, 12], Adomian decomposition method [13], Collocation method [14], and also Chen and et al. proposed a numerical method [15].

In this paper, we consider a system of the fractional differential equations, as follows:

$$
D_{*}^{\alpha_{i}} f_{i}(t)=g_{i}\left(t, f_{1}(t), f_{2}(t), \ldots, f_{n}(t)\right), \quad t \in[0, T], \quad i=1,2, \ldots, n,
$$

where $D_{*}^{\alpha_{i}} f_{i}(t)$ is derivative of order $\alpha_{i}$ for $f_{i}(t)$ and $0<\alpha_{i} \leq 1$. Thus for the system of Eq. (1), we consider the following initial condition:

$$
f_{i}(0)=y_{i}, \quad i=1,2, \ldots, n
$$

Note that $y_{1}, y_{2}, \ldots, y_{n}$ are constant numbers. There are several kinds of the fractional derivatives of a function [16, 17], but Rieman-Liouville and Caputo definitions are two of mostly used and famous for evaluating the fractional derivatives. In present paper, we use Caputo derivative.

The Caputo fractional derivative of order $0<\alpha<1$ is defined in the following form:

$$
D_{*}^{\alpha} y(t)=\frac{1}{\Gamma(1-\alpha)} \int_{0}^{t}(t-\tau)^{-\alpha} y^{\prime}(\tau) d \tau .
$$

Recall that the above formula for $\alpha=1$ coincides with the usual derivative operator of the first order.

We use The Müntz-Legendre polynomials [18, 19, 20] to approximate the solution of the Eq. (1). The main advantage of using the Muntz.Legendre polynomials is that their fractional derivative is still a Müntz-Legendre polynomial. Furthermore, approximate solutions of the fractional deferential equations with a collocation method based on the Müntz-Legendre polynomials show very good agreement with the exact solution. Another considerable advantage of the 
known polynomials in question is that the fractional derivatives of these polynomials can be expressed in terms of the same polynomials that are appropriate for solving the fractional differential equations.

A brief overview of the paper is as follows: first, the Jacobi polynomials are defined. Then, the definition of Müntz-Legendre polynomials and obtaining them via a stable recurrence relation are presented. After that, a method for evaluating the fractional derivatives of the Müntz-polynomials is introduced. Finally, the method of solving the fractional derivatives based on the Muntz.Legendre polynomials is explained and some numerical examples are given to demonstrate the effectiveness and accuracy of the proposed method.

\section{Preliminaries}

\subsection{Jacobi Polynomials}

The Jacobi polynomials are extensively used for solving fractional differential equations. They are orthogonal on the interval $[-1,1]$ with respect to the weight function $w^{(\alpha, \beta)}(t)=(1-t)^{\alpha}(1+t)^{\beta}$ where $\alpha, \beta>-1$. These polynomials can be obtained through the following recurrent relation:

$$
\begin{aligned}
J_{0}^{(\alpha, \beta)}(t)= & 1, \\
J_{1}^{(\alpha, \beta)}(t)= & \frac{1}{2}((\alpha-\beta)+(\alpha+\beta+2) t), \\
a_{k}^{(\alpha, \beta)} J_{k+1}^{(\alpha, \beta)}(t)= & b_{k}^{(\alpha, \beta)}(t) J_{k}^{(\alpha, \beta)}(t)-c_{k}^{(\alpha, \beta)} J_{k-1}^{(\alpha, \beta)}(t), \\
a_{k}^{(\alpha, \beta)}= & 2(k+1)(k+\alpha+\beta+1)(2 k+\alpha+\beta), \\
b_{k}^{(\alpha, \beta)}(t)= & (2 k+\alpha+\beta+1)((2 k+\alpha+\beta) \\
& \left.\times(2 k+\alpha+\beta+2) t+\alpha^{2}-\beta^{2}\right), \\
c_{k}^{(\alpha, \beta)}= & 2(k+\alpha)(k+\beta)(2 k+\alpha+\beta+2) .
\end{aligned}
$$

The initial derivative of the Jacobi polynomials can be obtained as follows:

$$
\frac{d}{d t} J_{k}^{(\alpha, \beta)}(t)=\frac{1}{2}(k+\alpha+\beta+1) J_{k-1}^{(\alpha+1, \beta+1)}(t)
$$




\subsection{Müntz-Legendre Polynomials}

Let $\Lambda_{n}=\left\{\lambda_{1}, \lambda_{2}, \ldots, \lambda_{n}\right\}$ be under condition $\operatorname{Re}\left(\lambda_{k}\right)>-\frac{1}{2}$ such that MüntzLegendre polynomials on the interval $(0,1]$ are defined as follows $[18,19]$ :

$$
L_{n}(t)=L\left(\Lambda_{n}, t\right)=\sum_{k=0}^{n} C_{n, k} t^{\lambda_{n}}, \quad C_{n, k}=\frac{\prod_{v=0}^{n-1}\left(\lambda_{k}+\bar{\lambda}_{v}+1\right)}{\prod_{v=0, v \neq k}^{n}\left(\lambda_{k}-\lambda_{v}\right)}
$$

Basic properties of the Müntz-Legendre polynomials:

$$
\begin{aligned}
& \left(L_{n}, L_{m}\right)=\int_{0}^{1} L_{n}(t) L_{m}(t) d t=\frac{\delta_{m n}}{\lambda_{n}+\bar{\lambda}_{n}+1}, \\
& L_{n}(1)=1, \\
& L_{n}^{\prime}(1)=\lambda_{n}+\sum_{k=0}^{n-1}\left(\lambda_{k}+\bar{\lambda}_{k}+1\right),
\end{aligned}
$$

Here, all $\lambda_{k}$ are chosen so that $\lambda_{k}=\alpha k$, the shifted Müntz-Legendre polynomials on the interval $I=[0, R)$ are defined as follows ( $\alpha$ is a positive real number):

$$
L_{I, n}(t: \alpha)=\sum_{k=0}^{n} C_{n, k}\left(\frac{t}{R}\right)^{\alpha k}, \quad C_{n, k}=\frac{(-1)^{n-k}}{\alpha^{n} k !(n-k) !} \prod_{v=0}^{n-1}((k+v) \alpha+1)
$$

Some of the shifted Müntz-Legendre polynomials' properties according to whatever has been said in [18] are:

$$
L_{I, n}(R: \alpha)=1, \quad L_{I, n}^{\prime}(R: \alpha)=\frac{\alpha n+\sum_{k=0}^{n-1}(2 \alpha k+1)}{R}
$$

Regarding Eq. (6), we have:

$$
\int_{0}^{R} L_{I, n}(t) L_{I, m}(t) d t=\frac{R \delta_{m n}}{1+2 \alpha n}
$$


Furthermore, following [14] a stable recurrence relation can be obtained for shifted Müntz-Legendre polynomials via the Jacobi polynomials, as follows:

$$
\begin{aligned}
& L_{I, 0}(t: \alpha)=1, \quad L_{I, 1}(t: \alpha)=\left(\frac{1}{\alpha}+1\right)\left(\frac{t}{R}\right)^{\alpha}-\frac{1}{\alpha}, \\
& a_{I, n} L_{I, n+1}(t: \alpha)=b_{I, n}(t) L_{I, n}(t: \alpha)-c_{I, n} L_{I, n-1}(t: \alpha), \\
& a_{I, n}=a_{n}^{\left(0, \frac{1}{\alpha}-1\right)}, \quad b_{I, n}(t)=b_{n}^{\left(0, \frac{1}{\alpha}-1\right)}\left(2\left(\frac{t}{R}\right)^{\alpha}-1\right), \quad c_{I, n}=c_{n}^{\left(0, \frac{1}{\alpha}-1\right)}
\end{aligned}
$$

In addition, according to [19] fractional derivative of these polynomials can also be obtained with the following equation:

$$
D_{*}^{\alpha} L_{I, n}(t: \alpha)=\frac{1+\alpha n}{\alpha \Gamma(1-\alpha) R^{\alpha}} \int_{0}^{R}\left(1-\tau^{\frac{1}{\alpha}}\right)^{-\alpha} J_{n-1}^{\left(1, \frac{1}{\alpha}\right)}\left(2\left(\frac{t}{R}\right)^{\alpha} \tau-1\right) d \tau
$$

\section{Solution of the Fractional Differential Equations}

In this section, we assume that $\alpha_{1}=\alpha_{2}=\cdots=\alpha_{n}=\alpha$ then we present an approximate solution for the unknown fractional differential equations in Eq. (1) as follows:

$$
f_{i}(t) \simeq \hat{f}_{i}(t)=\sum_{j=0}^{N} c_{i j} L_{I, j}(t: \alpha), \quad i=1,2, \ldots, n
$$

In addition, the fractional derivative of the unknown functions can be found by:

$$
D_{*}^{\alpha} f_{i}(t) \simeq D_{*}^{\alpha} \hat{f}_{i}(t)=\sum_{j=0}^{N} c_{i j} D_{*}^{\alpha} L_{I, j}(t: \alpha), \quad i=1,2, \ldots, n,
$$

where $D_{*}^{\alpha} L_{I, j}(t: \alpha)$ can be computed by the proposed Eq. (9) for evaluating the fractional derivative of the Müntz-Legendre polynomials and for the initial condition, we have:

$$
f_{i}(0) \simeq \hat{f}_{i}(0)=\sum_{j=0}^{N} c_{i j} L_{I, j}(0: \alpha)=y_{i}, \quad i=1,2, \ldots, n
$$


in this case, a system of Eq. (1) with boundary conditions is transformed into the following relations,

$$
\left\{\begin{array}{l}
\sum_{j=0}^{N} c_{i j} D_{*}^{\alpha} L_{I, j}(t: \alpha)=g_{i}\left(t, \sum_{j=0}^{N} c_{1 j} L_{I, j}(t: \alpha), \ldots, \sum_{j=0}^{N} c_{n j} L_{I, j}(t: \alpha)\right) \\
\sum_{j=0}^{N} c_{i j} L_{I, j}(0: \alpha)=y_{i}, \quad i=1,2, \ldots, n, \quad t \in[0, R]
\end{array}\right.
$$

to find $\hat{f}_{i}(t)$, we should obtain coefficients $c_{i j}$, for this purpose, we should satisfy the points $\theta_{k}=\frac{R}{2}-\frac{R}{2} \cos \left(\frac{\pi k}{N}\right), k=1,2, \ldots, N$ into above system of Eq. (13), and we have,

$$
\left\{\begin{array}{l}
\sum_{j=0}^{N} c_{i j} D_{*}^{\alpha} L_{I, j}\left(\theta_{k}: \alpha\right)=g_{i}\left(\theta_{k}, \sum_{j=0}^{N} c_{1 j} L_{I, j}\left(\theta_{k}: \alpha\right), \ldots, \sum_{j=0}^{N} c_{n j} L_{I, j}\left(\theta_{k}: \alpha\right)\right) \\
\sum_{j=0}^{N} c_{i j} L_{I, j}(0: \alpha)=y_{i}, \quad i=1,2, \ldots, n, \quad k=1,2, \ldots, N .
\end{array}\right.
$$

in turn, Eq. (14) produce an algebraic system of $n(N+1)$ equations for the $n(N+1)$ unknown coefficients $c_{i j}$. These coefficients are obtained by solving this system of algebraic equations with one of the known methods. Substituting them into Eq. (10) leads to an approximated solution of $f_{1}(t), f_{2}(t), \ldots, f_{n}(t)$.

\section{Numerical Examples}

Example 1. Consider the following system of the fractional deferential equations:

$$
\left\{\begin{array}{l}
D_{*}^{\alpha} f_{1}(t)-f_{1}(t)-f_{2}(t)=0 \\
D_{*}^{\alpha} f_{2}(t)+f_{1}(t)-f_{2}(t)=0, \quad t \in[0,2] \\
f_{1}(0)=0, \quad f_{2}(0)=1
\end{array}\right.
$$

The exact solution of these equations for $\alpha=1$ is given by $f_{1}(t)=e^{t} \sin (t)$ and $f_{2}(t)=e^{t} \cos (t)$. In Table 1 , we list the maximum absolute errors obtained by $f_{1}(t), f_{2}(t)$ with different values of $N$ and $\alpha=1$.

The approximate solutions obtained by $\hat{f}_{1}(t)$ and $\hat{f}_{2}(t)$ for different values of $\alpha$ and $N=30$ are plotted in Figure 1. 
Table 1: Maximum absolute error functions for Example 1

\begin{tabular}{cccccc}
\hline $\mathrm{N}$ & 10 & 15 & 20 & 25 & 30 \\
\hline $\max \left|f_{1}(t)-\hat{f}_{1}(t)\right|$ & $7.5 \times 10^{-8}$ & $1.2 \times 10^{-14}$ & $1.5 \times 10^{-21}$ & $4.3 \times 10^{-24}$ & $2.5 \times 10^{-27}$ \\
$\max \left|f_{2}(t)-\hat{f}_{2}(t)\right|$ & $7.6 \times 10^{-8}$ & $3.5 \times 10^{-14}$ & $1.8 \times 10^{-21}$ & $4.0 \times 10^{-24}$ & $2.3 \times 10^{-27}$ \\
\hline
\end{tabular}
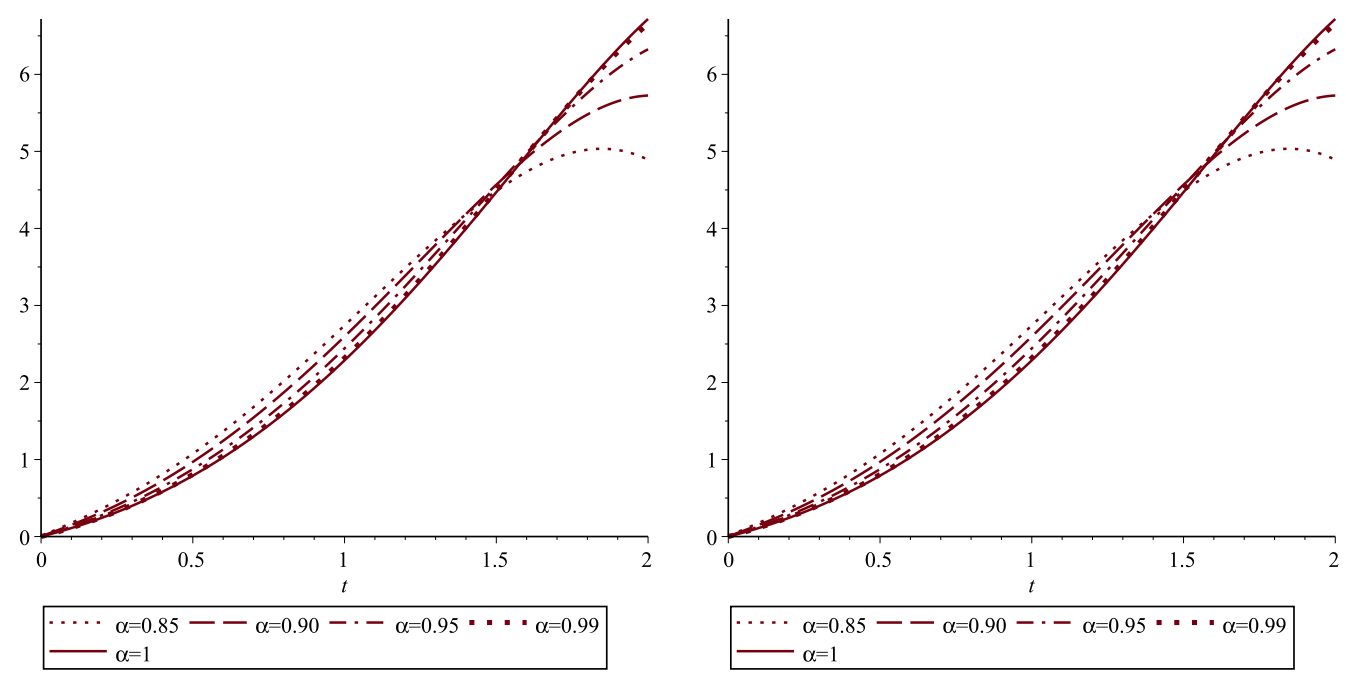

Figure 1: (a) The approximate solutions $f_{1}(t)$; (b) The approximate solutions $f_{2}(t)$.

Example 2. Consider the following system of the fractional deferential equations:

$$
\left\{\begin{array}{l}
D_{*}^{\alpha} f_{1}(t)=\frac{1}{2} f_{1}(t) \\
D_{*}^{\alpha} f_{2}(t)=\left(f_{1}(t)\right)^{2}+f_{2}(t), \quad t \in[0,2] \\
f_{1}(0)=1, \quad f_{2}(0)=0,
\end{array}\right.
$$

The exact solution of these equations for $\alpha=1$ is given by $f_{1}(t)=e^{t / 2}$ and $f_{2}(t)=t e^{t}$. In Table 2 , we list the maximum absolute errors obtained by $f_{1}(t)$, $f_{2}(t)$ with different values of $N$ and $\alpha=1$.

The approximate solutions obtained by $\hat{f}_{1}(t)$ and $\hat{f}_{2}(t)$ for different values of $\alpha$ and $N=30$ are plotted in Figure 2. 
Table 2: Maximum absolute error functions for Example 2

\begin{tabular}{cccccc}
\hline $\mathrm{N}$ & 10 & 15 & 20 & 25 & 30 \\
\hline $\max \left|f_{1}(t)-\hat{f}_{1}(t)\right|$ & $2.5 \times 10^{-13}$ & $4.1 \times 10^{-22}$ & $1.7 \times 10^{-31}$ & $3.0 \times 10^{-34}$ & $3.6 \times 10^{-37}$ \\
$\max \left|f_{2}(t)-\hat{f}_{2}(t)\right|$ & $3.1 \times 10^{-8}$ & $2.1 \times 10^{-15}$ & $3.2 \times 10^{-23}$ & $2.2 \times 10^{-25}$ & $2.3 \times 10^{-27}$ \\
\hline
\end{tabular}
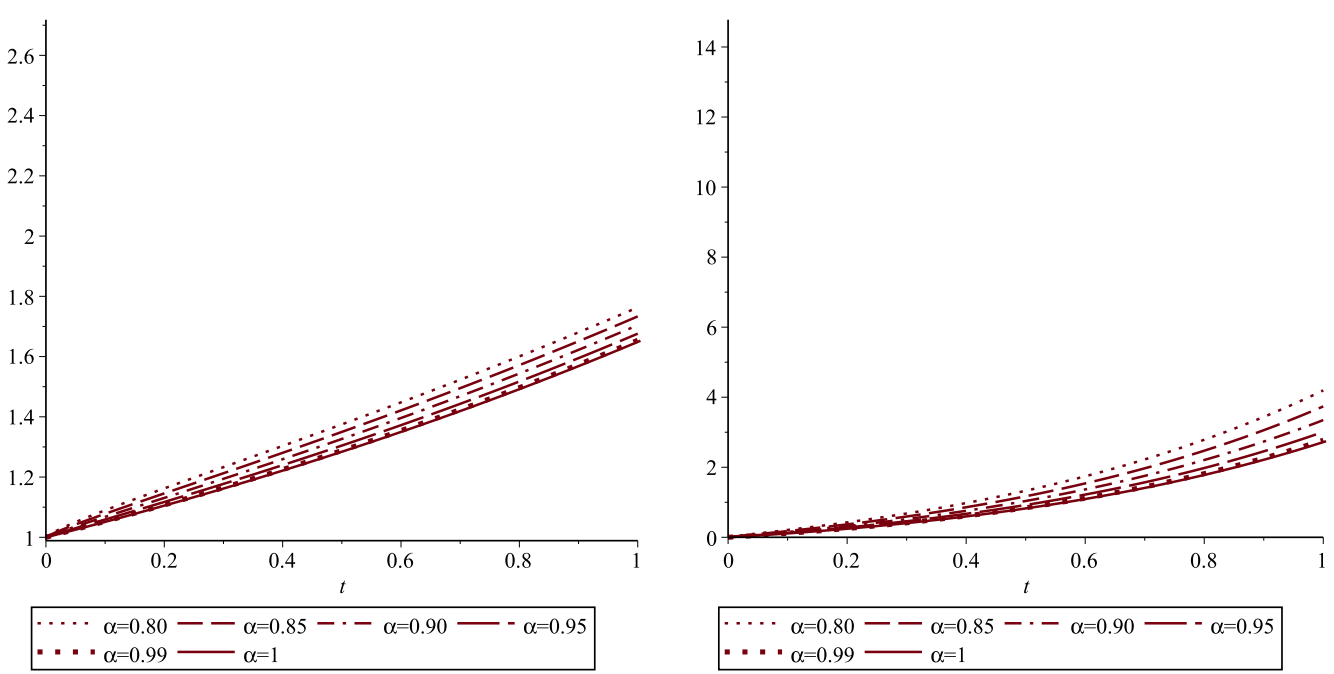

Figure 2: (a) The approximate solutions $f_{1}(t)$; (b) The approximate solutions $f_{2}(t)$.

\section{Conclusion}

In this paper, we presented a numerical method to obtain approximate solutions of the fractional deferential equations with a collocation method based on the Müntz-Legendre polynomials. The Müntz-Legendre polynomials are characterized for obtaining exact solutions of the fractional deferential equations. This is truly visible in results obtained through the numerical examples which their exact solutions are available.

\section{References}

[1] M. Ghasemi, M. Tavassoli Kajani, Numerical solution of time-varying delay systems by Chebyshev wavelets, Appl. Math. Model., 35 (2011), 5235-5244.

[2] M. Maleki, M. Tavassoli Kajani, Numerical approximations for Volterra's population growth model with fractional order via a multi-domain pseudospectral method, Appl. Math. Modl., 39 (2015), 4300-4308. 
[3] M. Tavassoli Kajani, M. Maleki, A. Kilicman, A multiple-step Legendre-Gauss collocation method for solving Volterra's population growth model, Math. Prob. Eng., 2013 (2013), $1-6$.

[4] M. Tavassoli Kajani, S. Vahdati, Z. Abbas, M. Maleki, Application of rational second kind Chebyshev functions for system of integro-differential equations on semi-infinite intervals, J. Appl. Math., 2012 (2012), 1-11.

[5] M. Tavassoli Kajani, M. Maleki, M. Allame, A numerical solution of Falkner-Skan equation via a shifted Chebyshev collocation method, AIP Conf. Proc., 1629 (2014), 381-386.

[6] D. Matignon,Stability results of fractional differential equations with applications to control processing, In: Proceeding of IMACS, IEEE-SMC, Lille, France (1996), 963-968.

[7] M.S. Tavazoei, M. Haeri, A note on the stability of fractional order systems, Math. Comput. Simul., 79 (2009), 1566-1576.

[8] Z.M. Odibat, Analytic study on linear systems of fractional differential equations, Comput. Math. Appl., 59 (2010), 1171-1183.

[9] V. Daftardar-Gejji, H. Jafari, Analysis of a system of nonautonomous fractional differential equations involving Caputo derivatives, J. Math. Anal. Appl., 328 (2007), 1026-1033.

[10] I. Hashim, O. Abdulaziz, S. Momani, Homotopy analysis method for fractional IVPs, Commun. Nonlinear Sci. Numer. Simul., 14 (2009), 674-684.

[11] M.M. Khader, Numerical treatment for solving the perturbed fractional PDEs using hybrid techniques, J. Comput. Phys., 250 (2013), 565-573.

[12] N.H. Sweilam, M.M. Khader, A.M. Nagy, Numerical solution of two-sided space-fractional wave equation using finite difference method, J. Comput. Appl. Math., 235 (2011), 28322841.

[13] H. Jafari, V. Daftardar-Gejji, Solving a system of nonlinear fractional differential equations using Adomian decomposition, J. Comput. Appl. Math., 196 (2006), 644-651.

[14] E.A. Rawashdeh, Numerical solution of fractional integro-differential equations by collocation method, Appl. Math. Comput., 176 (2006), 1-6.

[15] Y. Chen, X. Ke , Y. Wei, Numerical algorithm to solve system of nonlinear fractional differential equations based on wavelets method and the error analysis, Appl. Math. Comput., 251 (2015), 475-488.

[16] M. Caputo, Linear models of dissipation whose Q is almost frequency independent II, Geophys. J. Roy. Astronom. Soc., 13 (1967), 529-539.

[17] I. Podlubny, Fractional Differential Equations, Academic Press, NewYork, 1999.

[18] S. Esmaeili, M. Shamsi, Y. Luchko, Numerical solution of fractional differential equations with a collocation method based on Müntz polynomials, Comput. Math. Appl., 62 (2011), 918-929.

[19] P. Borwein, T. Erdélyi, J. Zhang, Müntz systems and orthogonal Müntz-Legendre polynomials, Trans. Amer. Math. Soc., 342, No. 2 (1994), 523-542.

[20] G.V. Milovanovic̀, Müntz orthogonal polynomials and their numerical evaluation, In: Applications and Computation of Orthogonal Polynomials, Ser. Numer. Math., 131, Birkhäuser, Basel (1999), 179-194. 
